\title{
Conservative Versus Tailored Surgical Treatment in Patients With First Time Lateral Patella Dislocation: a Randomized-controlled Trial
}

\section{Michael Liebensteiner}

Medical University of Innsbruck: Medizinische Universitat Innsbruck

Alexander Keiler ( $\nabla$ alexander.keiler@i-med.ac.at)

Medical University of Innsbruck: Medizinische Universitat Innsbruck https://orcid.org/0000-0003-1790296X

\section{Rene El Attal}

Feldkirch Hospital: Landeskrankenhaus Feldkirch

\section{Peter Balcarek}

ARCUS Sports Hospital: ARCUS Sportklinik

Florian Dirisamer

Orthopädie und Sportchirurgie Linz Puchenau

Johannes Giesinger

Medical University of Innsbruck: Medizinische Universitat Innsbruck

Gerd Seitlinger

Orthofocus Salzburg

Manfred Nelitz

MVZ Oberstdorf

Armin Keshmiri

MVZ im Helios, München

Jannik Frings

University Medical Center Hamburg-Eppendorf: Universitatsklinikum Hamburg-Eppendorf

Christoph Becher

ATOS Praxisklinik Heidelberg: ATOS Klinik Heidelberg GmbH \&amp; Co KG

\section{Paola Kappel}

Krankenhaus Koln-Merheim

\section{Daniel Wagner}

Hessingpark-Clinic GmbH Augsburg

\section{Geert Pagenstert}

Merian Iselin Department of Orthopedics and Surgery: Merian Iselin 
Keywords: Patella, instability, dislocation, conservative treatment, tailored surgical treatment

Posted Date: April 6th, 2021

DOI: https://doi.org/10.21203/rs.3.rs-382483/v1

License: (c) (1) This work is licensed under a Creative Commons Attribution 4.0 International License. Read Full License 


\section{Abstract}

\section{Background}

Patellar instability has a high incidence and occurs particularly in young and female patients. If the patella dislocates for the first time, treatment is usually conservative. However, this cautious approach carries the risk of recurrence and of secondary pathologies such as osteochondral fractures. An initial surgical treatment could possibly avoid these consequences of recurrent patella dislocation.

\section{Methods}

A prospective, randomized-controlled trial design is applied. Patients with unilateral first-time patella dislocation will be considered for participation. Study participants will be randomized to either conservative treatment or to a tailored patella stabilizing treatment. In the conservative group patients will use a knee brace and will be prescribed outpatient physical therapy. The surgical treatment will be performed in a tailored manner, addressing the pathologic anatomy that predisposes to patella dislocation.

The Banff Patellofemoral Instability-Instrument 2.0, recurrence rate, apprehension test, joint degeneration and the Patella Instability Severity Score will serve as outcome parameters. The main analysis will focus on the difference in change of the scores between the two groups within a two-year follow-up.

Statistical analysis will use linear mixed models. Power analysis was done for the comparison of the two study arms at 2-year follow-up with regard to the BPII score. A sample size of $\mathrm{N}=64$ per study arm (128 overall) provides $80 \%$ power (alpha $=0.05$, two-tailed) to detect a difference of 0.5 standard deviations in a t-test for independent samples.

\section{Discussion}

Although several studies have already dealt with this issue, there is still no consensus on the ideal treatment concept for primary patellar dislocation. Moreover, most of these studies show a unified surgical group, which means that all patients were treated with the same surgical procedure. This is regarded as a major limitation as surgical treatment of patella dislocation should depend on the patient's anatomic pathologies leading to patellar instability. To our knowledge, this is the first study investigating whether patients with primary patella dislocation are better treated conservatively or operatively with tailored surgery to stabilize the patella.

Trial registration

The study will be prospectively registered in the publicly accessible database www.clinicaltrials.gov.

\section{Background}


Instability of the patella has a high incidence, particularly in the young and female population. Because the vast majority of unstable patella are unstable towards latera/ and because instability is objective when the patella is fully dislocated the term "lateral patella dislocation (LPD)" is used throughout this study protocol.

First time or primary LPD is often treated conservatively. Although this is a cautious approach it bears risks of recurrence and secondary pathologies like osteochondral fractures. Therefore, it might well be speculated whether primary LPD should better be treated surgically. With this regard, several researchers already dealt with that issue (Only studies with Level of Evidence 1 or 2 were taken into account) (Table 1).

Apostolovic et al. performed a prospective, non-randomized controlled trial comparing 23 conservatively treated patients with 14 surgically treated patients in the context of primary LPD (1). The criterion to treat surgically was the presence of (osteo)chondral lesions. Besides loose body removal/refixation the patients received medial capsular repair and lateral retinacular release. The authors reported no differences between groups regarding recurrence rate or Cincinnati Knee Scores. Bitar et al. conducted a randomized-controlled trial on primary LPD (2). 39 patients were randomized to either non-operative treatment (immobilisation and physical therapy) or reconstruction of the medial patellofemoral ligament (MPFL). At 2 years follow-up the MPFL group was superior in terms of Kujala scores and recurrence rates. Camanho et al. also investigated patients with primary LPD (3). 33 patients were randomly assigned to either conservative treatment (immobilization and physiotherapy) or MPFL repair. The authors reported superior results in the surgical group. Christiansen et al. randomized 80 patients with primary LPD to either conservative therapy or MPFL repair (4). Patients were followed 2 years and demonstrated no significant differences between groups with regard to recurrence rate, KOOS score and Kujala Score. Two studies very similar to each other were published by Sillanpää et al. in 2008 and $2009(5,6)$. The older study investigated primary LPD in 61 military recruits. Conservative patients were compared to patients with MPFL repair, but were not randomized. The authors determined recurrence rates, Kujala Scores and "return to sports - rate" seven years postoperatively. While the surgical group was superior in terms of "return-to-sports" there were no significant differences in the other outcome parameters (5). The design of the other study from Sillanpää et al. was randomized-controlled. Again, the outcome parameters recurrence rates, Kujala Scores and "return to sports - rate" were determined in patients with conservative treatment and in those with surgery (medial repair or Roux-Goldthwait). Sillanpää et al stated that the patients treated surgically were superior in terms of recurrence rate but not in the other parameters (6). A randomized-controlled trial was also published by Petri et al. (7). In a multi-centric approach, patients with primary LPD were randomized to either conservative treatment or MPFL repair. Unfortunately, the trial was discontinued after 20 patients and did therefore not reach sufficient power.

In synopsis of the above-mentioned studies, there is no consensus on whether patients with first time LPD should be treated conservatively or surgically (Table 1). Besides, in most of those studies the surgically group was uniform, meaning that all patients were treated with the same surgical procedure. This is regarded as a major limitation as surgical treatment of LPD should be done in a tailored manner, 
depending on the patient's anatomic pathologies which lead to LPD. Moreover, it is striking that most above-mentioned studies applied MPFL repair. Only Bitar et al. performed MPFL-reconstruction.

Table 1. Current evidence on conservative vs. surgical treatment on patients with first-time lateral patella dislocation. Only studies with Level of Evidence 1 or 2 were taken into account.

\begin{tabular}{|c|c|c|c|c|c|c|c|c|c|}
\hline Author & Year & Random & $\begin{array}{c}\text { Evidence } \\
\text { Level }\end{array}$ & Surgical Procedure & $\begin{array}{l}\text { Outcome } \\
\text { Parameters }\end{array}$ & $\mathbf{n}$ & $\begin{array}{c}\text { mean FU } \\
\text { time }\end{array}$ & Findings & Misc \\
\hline Camanho & 2009 & $\mathrm{y}$ & 2 & MPFL repair & recurrence, Kujala & 33 & $3 y$ & $\begin{array}{l}\text { Surgery: better in } \\
\text { all parameters }\end{array}$ & $\begin{array}{l}\text { only 'statistical } \\
\text { trends' }\end{array}$ \\
\hline Bitar & 2012 & $y$ & 1 & MPFL recon & recurrence, Kujala & 41 & $44 \mathrm{mo}$ & $\begin{array}{l}\text { Surgery: signif. } \\
\text { better in all } \\
\text { parameters }\end{array}$ & \\
\hline Apostolovic & 2011 & $n$ & 2 & $\begin{array}{c}\text { loose body } \\
\text { removal/refixation, medial } \\
\text { capsular repair, lateral } \\
\text { retinacular release. }\end{array}$ & $\begin{array}{l}\text { Cincinatti Score, } \\
\text { Recurrence }\end{array}$ & 37 & $6 y$ & no diff & \\
\hline Christiansen & 2008 & $\mathrm{y}$ & 1 & MPFL repair & $\begin{array}{l}\text { recurrence, } \\
\text { Kujala, KOOS }\end{array}$ & 80 & $2 y$ & no diff & \\
\hline Petri & 2013 & $y$ & 1 & MPFL repair & $\begin{array}{l}\text { recurrence, } \\
\text { Kujala, } \\
\text { satisfaction }\end{array}$ & 20 & $2 y$ & no diff & Power $25 \%$ \\
\hline Sillanpää & 2008 & $n$ & 2 & MPFL repair & $\begin{array}{l}\text { recurrence, return } \\
\text { to sports, Kujala }\end{array}$ & 61 & $7 y$ & $\begin{array}{l}\text { Surg better return } \\
\text { to sports, rest: no } \\
\text { diff }\end{array}$ & \\
\hline Sillanpää & 2009 & $y$ & 1 & $\begin{array}{c}\text { medial repair or Roux- } \\
\text { Goldthwait }\end{array}$ & $\begin{array}{l}\text { recurrence, return } \\
\text { to sports, Kujala }\end{array}$ & 40 & $7 y$ & $\begin{array}{l}\text { Surgery better: } \\
\text { recurrence, other } \\
\text { parameters: no } \\
\text { diff }\end{array}$ & \\
\hline
\end{tabular}

Aims of the study

Due to the above-mentioned lack of consistent evidence it is the aim of the study to investigate whether patients with primary LPD are better treated conservatively or operatively (tailored surgery to stabilize the patella).

Hypotheses

It is hypothesized that patients with primary LPD when treated either conservatively or surgically (tailored stabilizing procedure) will show significant differences after two years in terms of the Banff Patellofemoral Instability-Instrument (BPII) 2.0 (Hypothesis 1). We also assume that the above-mentioned groups also differ significantly in terms of recurrent patella dislocations (Hypothesis 2 ).

\section{Methods}

Study design and participants 
A prospective, randomized-controlled trial design is applied. Before commencement of the study approval of the ethical committee (EC) was obtained (No. 1062/2020). Patients with objective, unilateral first time LPD will be considered for participation. After written informed consent the patients are included in the study. Excluded are a) patients with osteochondral lesions requiring removal/refixation, b) patients with recurrent $L P D, c)$ pregnant patients, d) patients $>45$ years of age and patients with physical maturity Kramer stage 1 to stage $3 a(8,9)$.

Clinical workup includes thorough history, physical evaluation, plain radiographs and magnetic resonance imaging in all patients. When the physical examination reveals suspicion of maltorsion syndrome, MRI (or CT scan) is done of the hip, knee and ankle to quantify femoral and tibial torsion. When the physical examination reveals suspicion of a relevant genu valgum or varum a whole leg radiograph is performed.

Patients successfully included in the study are then randomized to either conservative treatment or to a tailored patella stabilizing treatment. Block-Randomization is performed in advance with SPSS to guarantee equal group sizes. Syntax and seed for the random number generator are kept for reproducibility of the processes.

\section{Interventions}

In both groups in case of relevant hemarthrosis a joint aspiration if performed.

Conservative group

In the conservative group patients use a knee brace that a) protects the patella from lateralisation and $b$ ) limits knee range of motion. The range of motion limitation is set to 0-20-40 degrees for week 1 and 2, 010-60 degrees for week 3-4 and to 0-0-90 degrees for week 5-8. Partial weight-bearing is applied for week 1 and 2. Patients are prescribed outpatient physical therapy following a protocol suggested earlier by the "Patellofemoral Committee of the German-Speaking Arthroscopy Society (AGA)":

Phase 1 (week 1+2):

Range of motion $0-20-40^{\circ}$, partial weight-bearing

Phase 2 (week $3+4)$ :

Range of motion $0-10-60^{\circ}$, progression to full weight bearing, emphasis on quadriceps recruitment (especially vastus medialis).

Phase 3 (week 5-8):

Range of motion $0-0-90^{\circ}$, re-acquiring activities of daily living, core stability, sensorimotor training (leg axes stabilisation), strength training

Phase 4: 
Return to sports, dependent on the type and previous level of sports activity, gradual increase of training volume and intensity

Surgical group

The surgical treatment is performed in a tailored manner, addressing the respecting pathologic anatomy that predisposes to LPD. MPFL reconstruction is performed in every patient. Other surgical techniques listed below will be applied in individual combinations, dependent on patient's needs.

\section{MPFL reconstruction}

Reconstruction of the medial patellofemoral ligament (MPFL) is a proven technique for LPD and today's established standard treatment. However, some authors have reported a considerable complication rate (10). Many failures were reported due to inappropriate indications. The latter means performing isolated MPFL in patients with coexisting severe osseous pathologies like high-grade trochlear dysplasia or a pathologic tuberositas-tibiae-trochlea-groove distance (TT-TG distance) $(11,12)$. Isolated MPFL reconstruction is regarded as inappropriate in patients with: 1) TT-TG distance $>20 \mathrm{~mm}, 2$ ) femoral anteversion $\geq 40^{\circ}$ (n. Waidelich/Strecker), 3) high grade trochlea dysplasia, 4) severe patella alta, 5) tibiofemoral valgus $>5^{\circ}$. With accurate indications and surgical technique isolated MPFL reconstruction provides good outcome in patients with $\operatorname{LPD}(13,14)$. MPFL reconstruction is applied in all patients of the surgical group. MPFL reconstruction was reported with a high variety of surgical techniques (graft type, single vs. double bundle, type of fixation etc). The specific surgical technique is carried out on surgeon's preference at the respective center of the multicentric study.

Trochleoplasty

When the trochlea is flat or convex (dysplasia Dejour type B, C or D) a "deepening trochleoplasty" should be considered. The aim of the trochleoplasty is to A) reduce the too prominent anterior bone stock and B) create better conformity with the patella (concave groove) and a lateral trochlea facet as restraint against the lateralizing quadriceps pull. Many authors have reported that trochleoplasty leads to good clinical outcome in patients' LPD due to a dysplastic femoral trochlea (15-22).

Deepening trochleoplasty will be carried out in those patients of the surgical group who suffer from highgrade trochlea dysplasia.

\section{Tibial Tuberosity Transfer}

The most popular type of osteotomy in the setting of LPD is certainly the osteotomy and transfer of the tibial tuberosity (TTT). Many articles reported good clinical success for medialising TTT in patients with LPD and high TT-TG values (23-27). Similarly, good results were found for distalizing TTT in patients with LPD and patella alta $(28,29)$. TTT can be tailored to the pathology of the patient by performing combined medialization and distalization.

Medializing TTT will be applied to those patients with TT-TG distances $\geq 18 \mathrm{~mm}$ in the MRI. Distalizing TTT will be applied in patients with Caton-Deschamps Index $>1,2$ (30). 


\section{Derotational Osteotomy}

Derotational osteotomies of the femur (externally rotating) provide good results in patients with LPD and associated torsional deformities (31-33). However, the literature is incongruent on whether rotational osteotomies of the femur should be performed at the proximal or distal aspect (34-37). In the authors hand's derotational osteotomy is carried out at the distal femur.

In those patients with femoral antetorsion $\geq 40^{\circ}$ (n. Waidelich/Strecker) a distal femoral derotational osteotomy is carried out. The precise surgical technique for that procedure is given over to the surgeon of the respective center.

\section{Varus osteotomy}

In patients with valgus clinical appearance a weight-bearing whole leg radiograph should be performed to precisely assess the degree of the deformity in the frontal plane (mechanical femorotibial angle).

In cases with a mechanical femorotibial angle $>5$ degrees a varus osteotomy is performed at the location of the deformity.

Applying a "pragmatic" surgical approach, not each single pathology in the patient's anatomy is addressed. Instead, a maximum of 3 surgical techniques (including the MPFL reconstruction) are performed in one patient.

Outcome parameters

\section{Patient-reported outcome}

The Banff Patellofemoral Instability-Instrument (BPII) 2.0 was reported as valid, reliable and responsive patient-reported outcome tool in the field of patellofemoral instability $(38,39)$ and is used in the validated German version (40). The BPII 2.0 serves as one of two major outcome instruments (Hypothesis 1 ).

For exploratory reasons the following further patient-reported parameters will be assessed in both groups: As second disease specific score patients accomplish the Kujala Score (41) which was quoted as reliable, valid and responsive tool for patellofemoral disorders $(42,43)$. In addition, the Short-Form 12 is used (version 2, German; SF-12v2) (44) to determine the general health outcome and the Marx activity scale to rate a patient's physical activity (45). The Marx score asks for the highest activity in the last year. For postoperative monitoring of a patient's activity a "modified version of the Marx score" will be used that refers to the last 2 months.

All above-mentioned outcome scores are self-administered and will be assessed preoperatively, 6,12 months postoperatively and then yearly. Those scores are collected during routine visits at the hospital.

Other outcome parameters

Recurrence rate is assessed as second major outcome parameter (Hypothesis 2). To keep proper medical records on recurrent patella dislocations the patients are interviewed by telephone on a monthly basis (in 
addition to the above-mentioned visits at the hospital).

The apprehension test is assessed by an experienced observer during the above-mentioned routine clinical visits (Grade 0: no evasion, Grade 1: slight evasion/avoidance, Grade 2: gross evasion/avoidance, Grade 3: patient too anxious to allow the test).

Joint degeneration is assessed preoperatively and every three years postoperatively by means of MRI (PD-FSE with Fat-sat high-resolution in all three planes / T1-TSE, sagittal / T2 weighted, isotrope 3D sequence sagittal reformatted in all three planes). The semi-quantitative MRI Osteoarthritis Knee Score (MOAKS scoring) is applied to rate the degenerative changes determined by MRI (46). The MOAKS scoring is determined by always the same experienced musculoskeletal radiologist.

In addition, the Patella Instability Severity Score is assessed for exploratory reasons (47).

\section{Statistics}

Patient characteristics will be presented as means, standard deviations, and percentages. The main analysis will use linear mixed models that allow data modelling with a varying number of assessments per patient and time-varying covariates. Such a model will be used to compare the differences in changes over time between the two study groups. The following terms will be included in the model: a random baseline, a first-order autocorrelation covariance matrix, a fixed-effect patient group, a fixed effect time point, and the group-by-time interaction (reflecting the intervention effect). The BPII 2.0 will serve as the primary outcome parameters. The main analysis is "intention to treat" and will focus on the group difference in the change of BPII 2.0 scores between pre-op and 2-year follow-up. The above-mentioned secondary outcome parameters will be analysed with the same model.

Power / sample size analysis was done for the comparison of the two study arms at 2-year follow-up with regard to the BPII score. As there are no specific minimal important difference (MID) for the BPII available from the literature, we defined the MID to be 0.5 standard deviations following general recommendations from the literature (48). A sample size of $\mathrm{N}=64$ per study arm (128 overall) provides $80 \%$ power (alpha $=0.05$, two-tailed) to detect a difference of 0.5 standard deviations in a t-test for independent samples. To account for $20 \%$ attrition during the study period we plan to recruit 80 patients per study group (160 overall) at baseline.

\section{List Of Abbreviations}




\begin{tabular}{|ll|}
\hline 3D & three-dimensional \\
\hline AGA & Gesellschaft für Arthroskopie und Gelenkchirurgie \\
\hline BPII & Banff Patellofemoral Instability-Instrument \\
\hline CT & computer tomography \\
\hline EC & ethics committee \\
\hline ICF & informed consent form \\
\hline LPD & lateral patella dislocation \\
\hline MID & minimal important difference \\
\hline mm & millimeters \\
\hline MOAK & MRI Osteoarthritis Knee Score \\
\hline MPFL & medial patellofemoral ligament \\
\hline MRI & magnetic resonance imaging \\
\hline N & sample size \\
\hline PD-FSE & proton density-fast spin echo \\
\hline SF-12v2 & Short-Form 12 version 2 \\
\hline TSE & turbo spin echo \\
\hline TTT & transfer of the tibial tuberosity \\
\hline TT-TG distance & tuberositas-tibiae-trochlea-groove distance \\
\hline
\end{tabular}

\section{Declarations}

\section{Ethics approval and consent to participate}

Prior to study start, the study protocol and/or other appropriate documents were submitted to the ethics committee for approval. The study protocol was approved by the local ethics committee of the Medical University of Innsbruck (No. 1062/2020).

Every patient has to give his/her written consent before the participation in the clinical trial.

The content of the consent information is documented on the patient information/ informed consent form (ICF). The patient will be notified, if essential findings appear during the study.

\section{Consent for publication}


For any individual person's data in any form (including any individual details, images or videos), consent for publication must be obtained from that person, or in the case of children, their parent or legal guardian.

\section{Availability of data and materials}

The datasets used and/or analysed during the current study are available from the corresponding author on reasonable request.

\section{Competing interests}

The authors declare that they have no competing interests.

\section{Funding}

Article-processing charges are funded by the AGA - Gesellschaft für Arthroskopie und Gelenkchirurgie, Seefeldstrasse 123, 8034 Zürich, Switzerland.

\section{Authors' contributions}

All authors were involved in the drafting of the study protocol.

\section{Acknowledgements}

Not applicable.

\section{References}

1. Apostolovic M, Vukomanovic B, Slavkovic N, Vuckovic V, Vukcevic M, Djuricic G, et al. Acute patellar dislocation in adolescents: Operative versus nonoperative treatment. Int Orthop [Internet]. 2011 Oct [cited 2020 Oct 5];35(10):1483-7. Available from: https://pubmed.ncbi.nlm.nih.gov/21574051/

2. Bitar AC, Demange MK, D’Elia CO, Camanho GL. Traumatic patellar dislocation: Nonoperative treatment compared with MPFL reconstruction using patellar tendon. Am J Sports Med [Internet]. 2012 Jan [cited 2020 Oct 5];40(1):114-22. Available from: https://pubmed.ncbi.nlm.nih.gov/22016458/

3. Camanho GL, Viegas A de C, Bitar AC, Demange MK, Hernandez AJ. Conservative Versus Surgical Treatment for Repair of the Medial Patellofemoral Ligament in Acute Dislocations of the Patella. 
Arthrosc - J Arthrosc Relat Surg [Internet]. 2009 Jun [cited 2020 Oct 5];25(6):620-5. Available from: https://pubmed.ncbi.nlm.nih.gov/19501292/

4. Christiansen SE, Jakobsen BW, Lund B, Lind M. Isolated Repair of the Medial Patellofemoral Ligament in Primary Dislocation of the Patella: A Prospective Randomized Study. Arthrosc - $\mathrm{J}$ Arthrosc Relat Surg [Internet]. 2008 Aug [cited 2020 Oct 5];24(8):881-7. Available from: https://pubmed.ncbi.nlm.nih.gov/18657736/

5. Sillanpää PJ, Mäenpää HM, Mattila VM, Visuri T, Pihlajamäki H. Arthroscopic surgery for primary traumatic patellar dislocation: A prospective, nonrandomized study comparing patients treated with and without acute arthroscopic stabilization with a median 7-year follow-up. Am J Sports Med [Internet]. 2008 Dec [cited 2020 Oct 5];36(12):2301-9. Available from: https://pubmed.ncbi.nlm.nih.gov/18762668/

6. Sillanpää PJ, M.mattila V, Mäenpää H, Kiuru M, Visuri T, Pihlajamäki H. Treatment with and without initial stabilizing surgery for primary traumatic patellar dislocation: A prospective randomized study. J Bone Jt Surg - Ser A [Internet]. 2009 Feb 1 [cited 2020 Oct 5];91(2):263-73. Available from: https://pubmed.ncbi.nlm.nih.gov/19181969/

7. Petri M, Liodakis E, Hofmeister M, Despang FJ, Maier M, Balcarek P, et al. Operative vs conservative treatment of traumatic patellar dislocation: Results of a prospective randomized controlled clinical trial. Arch Orthop Trauma Surg [Internet]. 2013 Feb [cited 2020 Oct 5];133(2):209-13. Available from: https://pubmed.ncbi.nlm.nih.gov/23138693/

8. Alexander Krämer J, Schmidt S, Jü rgens K-U, Lentschig M, Schmeling A, Vieth V. The use of magnetic resonance imaging to examine ossification of the proximal tibial epiphysis for forensic age estimation in living individuals.

9. Krämer JA, Schmidt S, Jürgens KU, Lentschig M, Schmeling A, Vieth V. Forensic age estimation in living individuals using 3.0T MRI of the distal femur. Int J Legal Med [Internet]. 2014 [cited 2020 Jun 22];128(3):509-14. Available from: https://pubmed.ncbi.nlm.nih.gov/24504560/

10. Shah JN, Howard JS, Flanigan DC, Brophy RH, Carey JL, Lattermann C. A systematic review of complications and failures associated with medial patellofemoral ligament reconstruction for recurrent patellar dislocation [Internet]. Vol. 40, American Journal of Sports Medicine. SAGE PublicationsSage CA: Los Angeles, CA; 2012 [cited 2020 Oct 5]. p. 1916-23. Available from: http://journals.sagepub.com/doi/10.1177/0363546512442330

11. Hopper GP, Leach WJ, Rooney BP, Walker CR, Blyth MJ. Does degree of trochlear dysplasia and position of femoral tunnel influence outcome after medial patellofemoral ligament reconstruction? Am J Sports Med [Internet]. 2014 Mar [cited 2020 Oct 5];42(3):716-22. Available from: https://pubmed.ncbi.nlm.nih.gov/24458241/

12. Wagner D, Pfalzer F, Hingelbaum S, Huth J, Mauch F, Bauer G. The influence of risk factors on clinical outcomes following anatomical medial patellofemoral ligament (MPFL) reconstruction using the gracilis tendon. Knee Surgery, Sport Traumatol Arthrosc [Internet]. 2013 [cited 2020 Oct 5];21(2):31824. Available from: https://pubmed.ncbi.nlm.nih.gov/22538501/ 
13. Mackay ND, Smith NA, Parsons N, Spalding T, Thompson P, Sprowson AP. Medial patellofemoral ligament reconstruction for patellar dislocation: A systematic review. Orthop J Sport Med [Internet]. 2014 [cited 2020 Oct 5];2(8). Available from: https://pubmed.ncbi.nlm.nih.gov/26535352/

14. Stupay KL, Swart E, Shubin Stein BE. Widespread Implementation of Medial Patellofemoral Ligament Reconstruction for Recurrent Patellar Instability Maintains Functional Outcomes at Midterm to LongTerm Follow-up while Decreasing Complication Rates: A Systematic Review [Internet]. Vol. 31, Arthroscopy - Journal of Arthroscopic and Related Surgery. W.B. Saunders; 2015 [cited 2020 Oct 5]. p. 1372-80. Available from: https://pubmed.ncbi.nlm.nih.gov/25703288/

15. Neumann M V., Stalder M, Schuster AJ. Reconstructive surgery for patellofemoral joint incongruency. Knee Surgery, Sport Traumatol Arthrosc [Internet]. 2016 Mar 1 [cited 2020 Oct 5];24(3):873-8. Available from: https://pubmed.ncbi.nlm.nih.gov/25358690/

16. Banke IJ, Kohn LM, Meidinger G, Otto A, Hensler D, Beitzel K, et al. Combined trochleoplasty and MPFL reconstruction for treatment of chronic patellofemoral instability: a prospective minimum 2year follow-up study. Knee Surgery, Sport Traumatol Arthrosc [Internet]. 2014 Oct 21 [cited 2020 Oct 5];22(11):2591-8. Available from: https://pubmed.ncbi.nlm.nih.gov/23851967/

17. Dejour D, Byn P, Ntagiopoulos PG. The Lyon's sulcus-deepening trochleoplasty in previous unsuccessful patellofemoral surgery. Int Orthop [Internet]. 2013 [cited 2020 Oct 5];37(3):433-9. Available from: https://pubmed.ncbi.nlm.nih.gov/23275080/

18. Thaunat M, Bessiere C, Pujol N, Boisrenoult P, Beaufils P. Recession wedge trochleoplasty as an additional procedure in the surgical treatment of patellar instability with major trochlear dysplasia: Early results. Orthop Traumatol Surg Res [Internet]. 2011 Dec [cited 2020 Oct 5];97(8):833-45. Available from: https://pubmed.ncbi.nlm.nih.gov/22112463/

19. Utting MR, Mulford JS, Eldridge JDJ. A prospective evaluation of trochleoplasty for the treatment of patellofemoral dislocation and instability. J Bone Jt Surg - Ser B [Internet]. 2008 Feb [cited 2020 Oct 5];90(2):180-5. Available from: https://pubmed.ncbi.nlm.nih.gov/18256084/

20. Blønd L, Haugegaard M. Combined arthroscopic deepening trochleoplasty and reconstruction of the medial patellofemoral ligament for patients with recurrent patella dislocation and trochlear dysplasia. Knee Surgery, Sport Traumatol Arthrosc [Internet]. 2014 Oct 1 [cited 2020 Oct 5];22(10):2484-90. Available from: https://pubmed.ncbi.nlm.nih.gov/23370988/

21. Nelitz M, Dreyhaupt J, Lippacher S. Combined trochleoplasty and medial patellofemoral ligament reconstruction for recurrent patellar dislocations in severe trochlear dysplasia: A minimum 2-year follow-up study. Am J Sports Med [Internet]. 2013 May [cited 2020 Oct 5];41(5):1005-12. Available from: https://pubmed.ncbi.nIm.nih.gov/23467555/

22. Ntagiopoulos PG, Byn P, Dejour D. Midterm results of comprehensive surgical reconstruction including sulcus-deepening trochleoplasty in recurrent patellar dislocations with high-grade trochlear dysplasia. Am J Sports Med [Internet]. 2013 May [cited 2020 Oct 5];41(5):998-1004. Available from: https://pubmed.ncbi.nlm.nih.gov/23589587/ 
23. Longo UG, Rizzello G, Ciuffreda M, Loppini M, Baldari A, Maffulli N, et al. Elmslie-Trillat, Maquet, Fulkerson, Roux Goldthwait, and Other Distal Realignment Procedures for the Management of Patellar Dislocation: Systematic Review and Quantitative Synthesis of the Literature [Internet]. Vol. 32, Arthroscopy - Journal of Arthroscopic and Related Surgery. W.B. Saunders; 2016 [cited 2020 Oct 5]. p. 929-43. Available from: https://pubmed.ncbi.nlm.nih.gov/26921127/

24. Barber FA, McGarry JE. Elmslie-Trillat Procedure for the Treatment of Recurrent Patellar Instability. Arthrosc - J Arthrosc Relat Surg [Internet]. 2008 Jan [cited 2020 Oct 5];24(1):77-81. Available from: https://pubmed.ncbi.nlm.nih.gov/18182206/

25. Karataglis D, Green MA, Learmonth DJA. Functional outcome following modified Elmslie-Trillat procedure. Knee [Internet]. 2006 Dec [cited 2020 Oct 5];13(6):464-8. Available from: https://pubmed.ncbi.nlm.nih.gov/17011193/

26. Kumar A, Jones S, Bickerstaff DR, Smith TWD. Functional evaluation of the modified Elmslie-Trillat procedure for patello-femoral dysfunction. Knee [Internet]. 2001 [cited 2020 Oct 5];8(4):287-92. Available from: https://pubmed.ncbi.nlm.nih.gov/11706691/

27. Nakagawa K, Wada Y, Minamide M, Tsuchiya A, Moriya $H$. Deterioration of long-term clinical results after the Elmslie-Trillat procedure for dislocation of the patella. J Bone Jt Surg - Ser B [Internet]. 2002 [cited 2020 Oct 5];84(6):861-4. Available from: https://pubmed.ncbi.nlm.nih.gov/12211679/

28. Magnussen RA, De Simone V, Lustig S, Neyret P, Flanigan DC. Treatment of patella alta in patients with episodic patellar dislocation: a systematic review [Internet]. Vol. 22, Knee surgery, sports traumatology, arthroscopy : official journal of the ESSKA. Knee Surg Sports Traumatol Arthrosc; 2014 [cited 2020 Oct 5]. p. 2545-50. Available from: https://pubmed.ncbi.nlm.nih.gov/23392290/

29. Mayer C, Magnussen RA, Servien E, Demey G, Jacobi M, Neyret P, et al. Patellar tendon tenodesis in association with tibial tubercle distalization for the treatment of episodic patellar dislocation with patella alta. Am J Sports Med [Internet]. 2012 Feb [cited 2020 Oct 5];40(2):346-51. Available from: https://pubmed.ncbi.nlm.nih.gov/22109545/

30. Caton JH, Dejour D. Tibial tubercle osteotomy in patello-femoral instability and in patellar height abnormality. Int Orthop [Internet]. 2010 Feb [cited 2020 Oct 5];34(2 SPECIAL ISSUE):305-9. Available from: https://pubmed.ncbi.nlm.nih.gov/20066411/

31. Dickschas J, Harrer J, Pfefferkorn R, Strecker W. Operative treatment of patellofemoral maltracking with torsional osteotomy. Arch Orthop Trauma Surg [Internet]. 2012 Mar [cited 2020 Oct 5];132(3):289-98. Available from: https://pubmed.ncbi.nlm.nih.gov/21479863/

32. Nelitz M, Dreyhaupt J, Williams SRM, Dornacher D. Combined supracondylar femoral derotation osteotomy and patellofemoral ligament reconstruction for recurrent patellar dislocation and severe femoral anteversion syndrome: surgical technique and clinical outcome. Int Orthop [Internet]. 2015 Dec 1 [cited 2020 Oct 5];39(12):2355-62. Available from: https://pubmed.ncbi.nlm.nih.gov/26156717/

33. Strecker W, Dickschas J. [Torsional osteotomy: Operative treatment of patellofemoral maltracking]. Oper Orthop Traumatol. 2015 Dec;27(6):505-24. 
34. Bruce WD, Stevens PM. Surgical Correction of Miserable Malalignment Syndrome. J Pediatr Orthop [Internet]. 2004 [cited 2020 Oct 5];24(4):392-6. Available from:

https://pubmed.ncbi.nlm.nih.gov/15205621/

35. Delgado ED, Schoenecker PL, Rich MM, Capelli AM. Treatment of severe torsional malalignment syndrome. J Pediatr Orthop [Internet]. 1996 [cited 2020 Oct 5];16(4):484-8. Available from: https://pubmed.ncbi.nlm.nih.gov/8784702/

36. Dickschas J, Harrer J, Reuter B, Schwitulla J, Strecker W. Torsional osteotomies of the femur. J Orthop Res [Internet]. 2015 Mar 1 [cited 2020 Oct 5];33(3):318-24. Available from: https://pubmed.ncbi.nlm.nih.gov/25399673/

37. Stevens PM, Gililland JM, Anderson LA, Mickelson JB, Nielson J, Klatt JW. Success of torsional correction surgery after failed surgeries for patellofemoral pain and instability. Strateg Trauma Limb Reconstr [Internet]. 2014 [cited 2020 Oct 5];9(1):5-12. Available from: https://pubmed.ncbi.nlm.nih.gov/24338661/

38. Hiemstra LA, Kerslake S, Lafave M, Mohtadi NG. Concurrent Validation of the Banff Patella Instability Instrument to the Norwich Patellar Instability Score and the Kujala Score in Patients With Patellofemoral Instability. Orthop J Sport Med [Internet]. 2016 May 11 [cited 2020 Oct 5];4(5). Available from: https://pubmed.ncbi.nlm.nih.gov/27231700/

39. Lafave MR, Hiemstra L, Kerslake S. Factor Analysis and Item Reduction of the Banff Patella Instability Instrument (BPII). Am J Sports Med [Internet]. 2016 Aug 1 [cited 2020 Oct 5];44(8):2081-6. Available from: https://pubmed.ncbi.nlm.nih.gov/27166290/

40. Becher C, Attal R, Balcarek P, Dirisamer F, Liebensteiner M, Pagenstert G, et al. Successful adaption of the Banff Patella Instability Instrument (BPII) 2.0 into German. Knee Surgery, Sport Traumatol Arthrosc [Internet]. 2018 Sep 1 [cited 2020 Oct 5];26(9):2679-84. Available from: https://pubmed.ncbi.nlm.nih.gov/28785790/

41. Kujala UM, Jaakkola LH, Koskinen SK, Taimela S, Hurme M, Nelimarkka O. Scoring of patellofemoral disorders. Arthroscopy [Internet]. 1993 [cited 2020 Oct 5];9(2):159-63. Available from: https://pubmed.ncbi.nlm.nih.gov/8461073/

42. Crossley KM, Bennell KL, Cowan SM, Green S. Analysis of outcome measures for persons with patellofemoral pain: Which are reliable and valid? Arch Phys Med Rehabil [Internet]. 2004 May [cited 2020 Oct 5];85(5):815-22. Available from: https://pubmed.ncbi.nlm.nih.gov/15129407/

43. Watson CJ, Propps M, Ratner J, Zeigler DL, Horton P, Smith SS. Reliability and responsiveness of the lower extremity functional scale and the anterior knee pain scale in patients with anterior knee pain. J Orthop Sports Phys Ther [Internet]. 2005 [cited 2020 Oct 5];35(3):136-46. Available from: https://pubmed.ncbi.nlm.nih.gov/15839307/

44. Ware JE, Kosinski M, Keller SD. A 12-Item Short-Form Health Survey: Construction of Scales and Preliminary Tests of Reliability and Validity. Med Care [Internet]. 1996 [cited 2020 Oct 5];34(3):22033. Available from: https://pubmed.ncbi.nlm.nih.gov/8628042/ 
45. Marx RG, Stump TJ, Jones EC, Wickiewicz TL, Warren RF. Development and evaluation of an activity rating scale for disorders of the knee. Am J Sports Med [Internet]. 2001 [cited 2020 Oct 5];29(2):2138. Available from: https://pubmed.ncbi.nlm.nih.gov/11292048/

46. Hunter DJ, Guermazi A, Lo GH, Grainger AJ, Conaghan PG, Boudreau RM, et al. Evolution of semiquantitative whole joint assessment of knee OA: MOAKS (MRI Osteoarthritis Knee Score). Osteoarthr Cartil [Internet]. 2011 Aug [cited 2020 Oct 5];19(8):990-1002. Available from: https://pubmed.ncbi.nlm.nih.gov/21645627/

47. Balcarek P, Oberthür S, Hopfensitz S, Frosch S, Walde TA, Wachowski MM, et al. Which patellae are likely to redislocate? Knee Surgery, Sport Traumatol Arthrosc [Internet]. 2014 Sep 1 [cited 2020 Oct 5];22(10):2308-14. Available from: https://pubmed.ncbi.nlm.nih.gov/24005331/

48. Norman GR, Sloan JA, Wyrwich KW. Interpretation of Changes in Health-related Quality of Life. Med Care [Internet]. 2003 May [cited 2020 Oct 5];41(5):582-92. Available from: https://pubmed.ncbi.nlm.nih.gov/12719681/ 